\title{
What is the current orientation of undergraduate medical education in Korea?
}

\author{
Do-Hwan Kim, Eun Jeong Kim, Jinyoung Hwang, Jwa-Seop Shin and Seunghee Lee
}

Department of Medical Education, Seoul National University College of Medicine, Seoul, Korea

\section{현재 한국 기본의학교육은 어떤 졸업생을 양성하고자 하는가?}

서울대학교 의과대학 의학교육학교실

\section{김도환, 김은정, 황진영, 신좌섭, 이승희}

Purpose: The educational purpose of a medical school is important, because it guides educational decisions in an individual organization and projects the image of the doctors that we are generating. By analyzing the educational goals of entire medical schools, this study aimed to examine the current orientation and future direction of undergraduate medical education in Korea. Methods: Educational goals were collected from the website of each medical school and subjected to inductive content analysis. After identifying categories and themes, we examined the differences between medical school subgroups and compared the categories with competencies that have been suggested by the "Korean Doctor's Role."

Results: Thirteen themes were identified: medical expertise, professionalism, contribution to various levels of society, self-management and development, basic educational ideology, research ability, cooperation, leadership, dealing with future change, respect for life, creativity, problem-solving ability, and ability to educate. There was a significant difference in educational goals between medical schools when grouped by geographic location and affiliation of research-driven hospitals. Of the 16 competencies that are suggested by the Korean Doctor's Role, 12 had one or more corresponding categories.

Conclusion: Per their current educational purposes, Korean medical schools pursue a broad variety of competencies that need cultivating during the course of undergraduate medical education. Further research is needed to determine how best to apply these educational purposes in actual institutions and ultimately lead them to become part of the competency of a graduate.

Key Words: Educational goals, Educational purposes, Inductive content analysis, Korean doctor's role

\section{서론}

국가 및 인류 건강 증진은 의학, 특히 대학의학(academic medicine)이 궁극적으로 추구하는 지향점이라 할 수 있으며 [1], 의과대학은 이를 위해 교육이라는 역할을 주도적으로 수 행하고 있다. 시대와 사회의 요구에 부응할 수 있는 의사를 양 성하기 위해서 의과대학은 올바른 교육 지향을 보유할 필요
Received: January 29, 2015 • Revised: March 10, 2015 • Accepted: March 17, 2015 Corresponding Author: Seunghee Lee (http://orcid.org/0000-0001-8672-5253) Department of Medical Education, Seoul National University College of Medicine, 103 Daehak-ro, Jongno-gu, Seoul 110-799, Korea

Tel: +82.2.740.8406 Fax: +82.2.745.6373 email: 1shcho@snu.ac.kr
Korean J Med Educ 2015 Jun; 27(2): 87-98 http://dx.doi.org/10.3946/kjme.2015.27.2.87 eISSN: 2005-7288

(C) The Korean Society of Medical Education. All rights reserved. This is an open-access article distributed under the terms of the Creative Commons Attribution Non-Commercial License (http:// creativecommons.org/licenses/by-nc/3.0/), which permits unrestricted non-commercial use, distribution, and reproduction in any medium, provided the original work is properly cited. 
가 있는데, 조직이나 프로그램이 뛰어난 성과를 이루기 위해 서는 이론적 근거를 토대로 핵심 가치, 철학, 궁극적 목표가 무엇인지를 명확히 하는 것이 중요하기 때문이다[2]. 조직 또 는 프로그램이 지향하는 가치는 비전(vision), 미션(mission), 목적(goals), 목표(program objectives) 등으로 다양하게 일 컬어지며 이들 사이의 구분이 항상 명확한 것은 아니나[3], 대 체로 전자에서 후자로 갈수록 구체적이며, 측정 가능한 것을 기술하게 된다[2,3].

기존 연구에 따르면, 미국 의과대학들은 미션선언문(mission statement)을 통해 자신의 정체성과 교육의 목표를 표 명하고 있다[4]. 한편 국내 여러 의과대학은 교육목적 및 교육 목표(이하 교육목표)를 설정하고 이를 웹사이트에 게시하고 있는데, 기본적으로 교육목표는 의과대학에서 이루어지는 다 양한 교육영역의 의사결정에서 중요한 역할을 한다. 예컨대 정규 교육과정에 관한 주요 의사결정 시에 판단의 기준 및 핵 심 참고자료로서의 역할을 하며[5], 비정규 교육과정[6]이나 교수개발 프로그램[7]에서도 개별 의과대학의 고유한 교육목 표가 고려되어야 한다는 점이 논의된 바 있다. 이와 같은 교육 목표의 의과대학 조직 내적인 역할은 '조직의 비전을 제시하 고 전략기획을 수립(strategic planning)하기 위한 필수 문헌' 이라는 미션선언문의 의의와도 맞닿아 있다[8].

의과대학의 교육목표는 한국의학교육평가원(이하 의평원) 의 의과대학 인증평가 사업과 더불어 또 다른 중요성을 갖게 되었는데, 인증기준에 교육목표의 보유 여부 외에도 주기적 평가, 교육목표를 근거로 한 졸업성과 설정, 교육과정에의 반영 등이 평가 항목으로 포함되어 있기 때문이다[9]. 교육 목표가 이처럼 강조되는 것은 최근 국내 여러 의과대학이 성 과바탕교육으로의 이행단계에 있으며, 그 과정에서 기본의 학교육 성과의 뼈대인 교육목표의 재정립이 필요하기 때문 이기도 하다.

한편 국내 41 개 의과대학/의학전문대학원(이하 의전원) 전 체를 의사 양성이라는 동일한 기능을 수행하는 하나의 집합 체로 보았을 때, 개별 의과대학으로 나뉘어 교육을 받은 의과 대학생들이 미래에는 의사라는 단일한 전문직 집단의 구성원 이 될 것임을 쉽게 예상할 수 있다. 따라서 현재 기본의학교육 의 방향을 살펴보는 것은 미래 한국의 의사들이 어떠한 모습 일 것인가를 미리 점검해본다는 의의가 있다. 나아가 설립 주
체, 소재 지역, 학제, 학년별 정원 등 다양한 기준에 따라 의과 대학을 분류하고 교육 특성을 비교한다면 그 방향을 조금 더 구체적으로 알 수 있을 것이다. 또한 2013년 일부 의과대학 부속병원이 보건복지부로부터 연구중심병원으로 지정된 바 있는데[10], 부속병원의 이러한 차이가 의과대학 교육과도 연 관되어 나타날 수 있다.

마지막으로 국내 기본의학교육에서 교육목표가 가지는 의 의는 최근 한국의사협회에서 제작, 배포한 "2014 한국의 의사 상(이하 한국의 의사상)"[11]에서도 찾을 수 있다. "한국의 의 사상”은 포괄적 역량으로서 대한민국의 의사에게 기대되는 역할과 덕목을 다루고 있기 때문에, 이를 교육목표로 대변되 는 현재 기본의학교육의 방향과 비교하는 것은 교육과정 개 발의 첫 단계인 '일반적 요구사정(general needs assessment)' 에 비견될 수 있다[12]. 따라서 만약 이 둘 사이의 격차가 드 러난다면, 이를 기본의학교육 개선 목적으로 활용 가능할 뿐 아니라, 졸업 후 의학교육의 교육과정을 점검하고 개선하는 근거로도 활용할 수 있을 것이다.

교육목표의 여러 중요성에도 불구하고, 현재까지 국내 의 학교육과 관련하여 교육목표에 대한 연구는 거의 이루어진 바가 없으며, 범위를 넓혀 보아도 한국 상급종합병원의 미션 선언문에 대한 연구[13] 정도에 그치고 있다. 이에 본 연구에 서는 (1) 내용분석(content analysis)을 활용하여 41개 의과 대학/의전원의 교육목표를 종합적으로 분석한 뒤, (2) 설립주 체, 학년별 정원, 학제, 지리적 위치, 연구중심병원 보유 여부 등으로 의과대학의 하위집단을 구분하고, 이들 간 어떠한 교 육목표의 차이가 있는지 확인하고자 하였다. 마지막으로 (3) "한국의 의사상"에서 제시된 역량과의 비교를 통하여 현재 한 국 의과대학이 양성하는 의사가 사회적으로 요구되는 의사상 에 어느 정도 부합하는가를 살펴보고자 한다.

\section{대상 및 방법}

\section{1. 연구 대상 선정 및 자료 수집}

본 연구를 위하여 일차적으로 전국 41개 의과대학/의전원 의 공식 웹사이트에 ‘교육목표’ 또는 이에 상응하는 명칭의 웹 
페이지를 찾고, 포함되어 있는 자료를 모두 수집하였다. 이 과 정에서 '교육목적'이나 '교육목표' 외에도 해당 웹페이지에 함 께 포함된 ‘핵심가치', '교육이념' ‘졸업성과’ 등의 자료도 수집 되었다.

각 의과대학의 공식 웹사이트 접속을 위하여 한국의과대학 · 의학전문대학원협회 웹사이트에 공지된 주소를 참조하였으 며, 일부 주소에 오류가 있는 경우에는 Google 등의 개방형 검색엔진을 활용하여 접속하였다. 자료의 수집은 2014년 10 월 28일부터 11월 25일에 걸쳐서 이루어졌으며, 주기적 확인 을 통하여 자료 수집 기간 내에 웹사이트에 공지된 교육목표 의 변경이 있었던 경우에는 가장 최신의 교육목표를 재수집 하여 분석 대상으로 하였다.

\section{2. 분석 대상 정의}

'교육목표' 웹페이지 내에는 '교육목적'과 '교육목표' 외에도 다양한 자료가 함께 포함되어 있었다. 또한 '교육목적' 또는 ‘교육목표라 명시된 것들도 그 형식과 기술방식이 다양하였 기에 연구진은 Table 1 과 같은 기준에 따라 분석 대상을 정의 하였다.

\section{3. 교육목표 내용분석}

내용분석은 텍스트의 많은 단어를 적은 수의 카테고리로 줄
이는 체계적이고 재현가능한 연구 방법으로서, 크게 귀납적 접근법과 연역적 접근법이 가능하다. 이 중 귀납적 접근법은 개방코딩(open coding), 그룹화(grouping), 범주화(categorization), 추상화(abstraction)의 환원적 과정을 거쳐서 분석 대상을 설명할 수 있는 개념틀(conceptual system) 또는 개 념 범주(conceptual categories)를 도출하는 질적 연구방법 이다[14,15]. 본 연구에서 분석 절차는 내용분석의 방법론을 다룬 기존 문헌을 따랐으며[14,15], 그 외에 국내 의학교육 분 야에서 내용분석을 활용하여 수행된 연구를 참고하였다[16]. 모든 분석단계에서 분석 과정과 분석 결과에 대한 점검을 위 하여 연구자 간 수 차례 논의과정을 거쳤다.

\section{1) 1 단계 개방코딩}

본 내용분석에서는 교육목적 또는 교육목표에 나타나는 교 육의 지향을 의미단위로 하였다. 교육의 지향에는 '지식' '수 기'와 같이 비교적 구체적인 목표 외에도 '가치관', '태도'와 같 은 광범위하거나 추상적인 목표도 포함하였다. 일반적으로 한 문장 내에는 다수의 의미단위가 포함되어 있었고, 이들을 모두 개별 의미단위로 코딩시트(coding sheet)에 입력하였다. 개방코딩의 예시는 다음과 같다.

[원문] 의사로서 갖추어야 할 가치관과 태도 수기 및 지식을 습득하여 건강 증진, 질환의 예방과 치료 및 재활을 수행한다. [개방코딩] (1) 가치관, (2) 태도, (3) 수기, (4) 지식, (5) 건

Table 1. Inclusion and Exclusion Criteria for Selection of Subjects

\begin{tabular}{|c|c|c|}
\hline Types of the criteria & Inclusion criteria & Exclusion criteria \\
\hline Title & $\begin{array}{l}\text { 11. Any texts, tables, or figures titled 'Educational } \\
\text { goals' or 'Educational purposes }{ }^{\text {'al }}\end{array}$ & $\begin{array}{l}\text { E1. Any text, table, or figure not titled 'Educational } \\
\text { goals' or 'Educational purposes,' i.e., 'Core value,' } \\
\text { 'Ideals of education,' 'Exit outcome,' etc. }\end{array}$ \\
\hline Level of detail & $\begin{array}{l}\text { 12. If it has a multi-level hierarchical structure or } \\
\text { includes contents of the two highest levels }{ }^{\text {a }}\end{array}$ & $\begin{array}{l}\text { E2-1. If it has a multi-level hierarchical structure and } \\
\text { excludes contents of the third highest levels or lower. } \\
\text { E2-2. Excludes ancillary parts, such as 'additional } \\
\text { explanation,' 'annotations,' 'footnote,' etc. }\end{array}$ \\
\hline Target students & $\begin{array}{l}\text { I3. 'Educational goals' or 'Educational purposes' for } \\
\text { medical doctor training }\end{array}$ & $\begin{array}{l}\text { E3-1. 'Educational goals' or 'Educational purposes' for } \\
\text { colleges other than medical school, such as nursing } \\
\text { schools. } \\
\text { E3-2. 'Educational goals' or 'Educational purposes' for } \\
\text { general graduate school in a medical college. }\end{array}$ \\
\hline
\end{tabular}

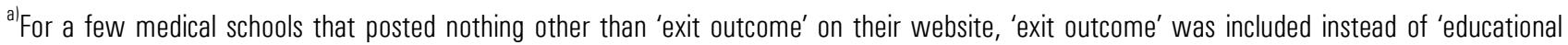
purposes' as a subject of analysis. However, in fairness to other medical schools, only outcomes of the two highest levels were included according to the criterion 12 . 
강증진수행, (6) 질환예방수행, (7) 질환치료수행, (8) 질환재 활수행

\section{2) 2 단계 그룹화}

2 단계에서는 1 단계에서 추출한 의미단위 중 의미가 동일하 나 서로 다르게 표현된 것들에 공통의 이름을 부여하였다. 이 단계에서는 가급적 원문의 표현을 유지하였고, 상이한 개념 들의 범주화는 최대한 지양하였다. 그룹화의 예시는 다음과 같다.

[예시 1] (1) 술기능력, (2) 의료수기, (3) 의학기능, (4) 의 학기본수기, (5) 의학기술습득 $\rightarrow$ '의학술기'로 명명

[예시 2] (1) 다방면 진출, (2) 다양한 전공분야, (3) 다양한 전문분야, (4) 다양한 진로 $\rightarrow$ '다양한 분야 진출로 명명

\section{3) 3 단계 범주화}

3 단계에서는 2 단계에서 명명된 그룹들을 분류하고, 함께 분류된 그룹을 대표할 수 있는 범주를 추출하는 작업을 수행 하였다. 이 과정에서는 개별 그룹에게 부여된 명칭과 더불어, 각 의미단위가 원문의 맥락에서 어떠한 취지로 서술되었는가 도 함께 고려하였다. 범주화의 예시는 다음과 같다.

[예시 1] (1) 도덕성, (2) 양심, (3) 윤리의식, (4) 정직 $\rightarrow$ ‘윤리 및 도덕성'으로 범주화

[예시 2] (1) 자기개발, (2) 자기주도학습, (3) 평생학습, (4) 최신지견습득 $\rightarrow$ '평생학습으로 범주화

\section{4) 4단계 추상화}

4단계에서는 3단계에서 도출된 여러 범주를 아우를 수 있 는 압축된 주제(theme)를 도출하고자 하였다. 3 단계와 마찬 가지로 4단계에서도 범주 자체의 명칭뿐 아니라, 이전 단계에 서 해당 범주가 도출되는 데 기여한 그룹의 이름, 의미단위, 원문의 맥락 등을 종합적으로 고려하였다.

\section{4. “한국의 의사상”과의 비교}

귀납적 내용분석을 통하여 도출한 범주를 "한국의 의사상" 을 대응시키는 작업은 연역적 내용분석의 접근법을 활용하였 다. 연구자가 수집한 자료로부터 새로운 범주를 도출하는 귀 납적 내용분석과 달리, 연역적 내용분석은 기존에 개념이나 모델이 개발되어 있는 경우에 활용할 수 있는 내용분석 방법 이다[14]. 연역적 내용분석에서 연구자는 기존 자료 및 문헌 을 근거로 자료를 대응시킬 범주와 하위 범주에 대한 표 (matrix)를 작성한 후, 이에 따라 연구자의 자료를 대응시키 는 과정을 밟게 된다[14]. "한국의 의사상"은 다섯 개의 영역 으로 이루어져 있으며, 각 영역은 2개에서 4 개의 역량을 포함 하여, 총 16 개의 역량을 제시하고 있다. 각 역량 아래에는 다 시 2 개에서 7 개의 세부 역량이 제시되어 있어, 총 65 개의 세 부 역량을 제시하고 있다. 본 연구에서는 앞서 귀납적 내용분 석으로 도출한 44 개의 범주를 “한국의 의사상”에 제시된 16 개 의 역량에 대응시키고자 하였으며, 범주가 역량에 대응하는 지 여부는 각 역량의 하위에 제시된 세부 역량을 기준으로 판 단하였다. 앞선 귀납적 내용분석과 마찬가지로 모든 분석단 계에서 분석 과정과 분석 결과에 대한 점검을 위하여 연구자 간 수 차례 논의과정을 거쳤다.

\section{5. 통계분석}

내용분석을 통해 도출한 의미단위, 범주, 주제 등의 빈도에 대한 정량적 분석에는 SPSS version 20.0 통계프로그램 (IBM Corp., Armonk, USA)을 사용하였다. 다양한 의과대 학 하위집단 간 차이를 비교하기 위해 기술통계분석, Student t-test, chi-square test를 실시하였다. 통계적 유의성은 p< 0.05 수준에서 측정하였다.

\section{결과}

\section{1. 기술통계량}

41개 의과대학/의전원의 교육목표와 교육목적으로부터 추 출한 의미단위는 총 842 개였으며, 제일 적은 수의 의미단위를 가진 학교는 6개, 가장 많은 수의 의미단위를 가진 학교는 39 개로 한 학교당 평균 20.54개의 의미단위를 포함하고 있었다 (Table 2). 이는 그룹화 후 평균 16.68 개, 범주화 후 평균 14.71 개, 추상화 후 평균 8.29개로 점진적으로 정리되었다. 최종적으로 도출된 13 개의 주제 중 가장 많은 주제를 포함한 학교는 13 개 주제를 모두 포함하고 있었으며, 가장 적은 학교 는 4개 주제만을 포함하고 있었다. 
Table 2. Descriptive Statistics of the Meaning Units, Groups, Categories, and Themes after Conducting Inductive Content Analysis

\begin{tabular}{lcccc}
\hline \multicolumn{1}{c}{ Item } & Meaning units & Groups & Categories & Themes \\
\hline Total no. & 842 & 89 & 44 & 13 \\
Medical schools & & & & \\
Mean \pm SD & $20.54 \pm 7.45$ & $16.68 \pm 4.97$ & $14.71 \pm 3.94$ & $8.29 \pm 2.04$ \\
Minimum & 6 & 6 & 6 & 4 \\
Maximum & 39 & 27 & 23 & 13 \\
\hline
\end{tabular}

SD: Standard deviation.

Table 3. Identified Themes and Categories

\begin{tabular}{|c|c|c|c|c|}
\hline No. & Themes & $\mathrm{n}^{\text {al }}$ & Categories & $n^{b /}$ \\
\hline \multirow[t]{6}{*}{1} & \multirow[t]{6}{*}{ Medical expertise } & \multirow[t]{6}{*}{40} & Biomedical knowledge & 33 \\
\hline & & & Medical skills & 30 \\
\hline & & & Primary healthcare & 18 \\
\hline & & & Ability to making a diagnosis and provide treatment & 17 \\
\hline & & & Expertise & 12 \\
\hline & & & Health promotion & 10 \\
\hline \multirow[t]{9}{*}{2} & \multirow[t]{9}{*}{ Professionalism } & \multirow[t]{9}{*}{38} & Ethics and morality & 31 \\
\hline & & & Attitudes & 14 \\
\hline & & & Personality & 13 \\
\hline & & & Sense of duty & 11 \\
\hline & & & Social accountability & 10 \\
\hline & & & Being cultured (humanities and social sciences) & 6 \\
\hline & & & Values & 5 \\
\hline & & & Professionalism & 4 \\
\hline & & & Wisdom & 1 \\
\hline \multirow[t]{5}{*}{3} & \multirow{5}{*}{$\begin{array}{l}\text { Contribution to various levels of } \\
\text { society }\end{array}$} & \multirow[t]{5}{*}{36} & Community-level contribution & 24 \\
\hline & & & National-level contribution & 22 \\
\hline & & & Human-kind-level contribution & 21 \\
\hline & & & Service spirit & 17 \\
\hline & & & International-level contribution & 13 \\
\hline \multirow[t]{5}{*}{4} & \multirow[t]{5}{*}{ Self-management and development } & \multirow[t]{5}{*}{35} & Lifelong learning & 34 \\
\hline & & & Advance towards various areas of medicine & 10 \\
\hline & & & Self-reflection & 9 \\
\hline & & & Good physical health & 1 \\
\hline & & & Self-realization & 1 \\
\hline \multirow[t]{2}{*}{5} & \multirow[t]{2}{*}{ Basic educational ideology } & \multirow[t]{2}{*}{28} & Founding philosophy/ideals of education & 19 \\
\hline & & & Religious spirit & 10 \\
\hline 6 & Research ability & 28 & Research ability & 28 \\
\hline \multirow[t]{6}{*}{7} & \multirow[t]{6}{*}{ Cooperation } & \multirow[t]{6}{*}{26} & Patient-centered care & 17 \\
\hline & & & Respect for diversity & 10 \\
\hline & & & Cooperation & 9 \\
\hline & & & Communication & 8 \\
\hline & & & Teamwork among health professions & 7 \\
\hline & & & Trust & 3 \\
\hline \multirow[t]{2}{*}{8} & \multirow[t]{2}{*}{ Leadership } & \multirow[t]{2}{*}{24} & Leadership & 24 \\
\hline & & & Healthcare manager & 2 \\
\hline
\end{tabular}


Table 3. (Continued)

\begin{tabular}{|c|c|c|c|c|}
\hline No. & Themes & $n^{a l}$ & Categories & $n^{\text {bl }}$ \\
\hline \multirow[t]{2}{*}{9} & Dealing with future change & 20 & Cope actively with changes & 14 \\
\hline & & & Future-oriented & 8 \\
\hline \multirow[t]{2}{*}{10} & Respect for life & 20 & Respect for life & 15 \\
\hline & & & Love for mankind & 12 \\
\hline 11 & Creativity & 20 & Creativity & 20 \\
\hline \multirow[t]{2}{*}{12} & Problem-solving ability & 19 & Problem-solving ability & 17 \\
\hline & & & Reasoning ability & 7 \\
\hline 13 & Ability of education & 6 & Ability of education & 6 \\
\hline
\end{tabular}

${ }^{a}$ The number of medical schools that contains the theme in their educational goals or purposes, ${ }^{b}$ The number of medical schools that contains the category in their educational goals or purposes.

\section{2. 교육목적 및 교육목표의 범주와 주제}

귀납적 내용분석과정을 거쳐 41개 의과대학/의전원의 교육 목적과 교육목표로부터 최종적으로 44 개의 범주와 13 개의 주 제를 도출하였다(Table 3). 가장 많은 수의 의과대학 교육목 표에 포함되어 있었던 주제는 ‘의(醫) 전문성’이었으며, 가장 적은 수의 의과대학 교육목표에 포함되어 있었던 주제는 '교 육능력'이었다. 13 개의 주제를 빈도 순으로 살펴보면 '의(醫) 전문성, ‘전문직업성', ‘사회 여러 층에 대한 기여', ‘자기관리 및 개발, '교육기본이념', '연구, '협력', '리더십', '생명존중', '미래-변화 대처', '창의성, '문제해결능력, '교육능력'과 같다.

각 범주는 최소 1 개 의과대학, 최대 34 개 의과대학 교육목 표에 포함되어 있었다. 가장 높은 빈도로 등장한 다섯 개 범주 는 '평생학습, ‘의과학지식', ‘윤리 및 도덕성', ‘의학술기', '연구 능력'이었다. 13 개 주제는 최소 1 개, 최대 9 개의 범주로 구성 되었으며, 각 주제에 포함된 범주를 살펴보면 다음과 같다. '의(醫) 전문성'은 6개(건강증진, 의과학지식, 의학술기, 일차 의료, 전문성, 진료능력), '전문직업성'은 9개(가치관, 사명감, 사회적 책무성, 윤리 및 도덕성, 인격, 인문사회학적 소양, 전 문직업성, 지혜, 태도), '사회 여러 층에 대한 기여'는 5 개(지역 사회수준기여, 국가수준기여, 인류수준기여, 봉사정신, 국제 수준기여), '자기관리 및 개발은 5개(다양한 분야 진출, 신체 적 건강, 자기성찰능력, 자아실현, 평생학습), '교육기본이념' 은 2개(건학이념/교육이념, 신앙정신), '연구능력'은 1 개(연구 능력), '협력'은 6개(보건의료인간협력, 상호다양성존중, 신뢰, 의사소통, 협력, 환자중심진료), '리더십'은 2개(리더십, 보건
의료관리자), '미래-변화 대처'는 2개(능동적 변화 대처, 미래 지향), '생명존중'은 2개(생명존중, 인류애), '창의성'은 1개(창 의성), '문제해결능력'은 2 개(문제해결능력, 추론능력), '교육 능력은 1 개(교육능력)의 범주를 포함하였다.

\section{3. 대학 유형별 비교}

41 개 의과대학/의전원을 설립 주체, 학년별 정원, 학제, 지 리적 위치, 연구중심병원 보유 여부 등에 따라 구분하였으며, 하위집단별로 교육목적 및 교육목표에 어떠한 차이가 있는가 를 살펴보았다. 설립 주체는 국립과 사립으로, 학년별 정원은 50 명을 기준으로, 지리적 위치는 의과대학이 소재한 지역을 기준으로 구분하였다. 학제는 부분적 도입과 전면적 도입을 구분하지 않고 의전원 도입 여부를 기준으로 하였으며, 연구 중심병원 보유 여부는 해당 웹사이트[10]에 공지된 정보를 기 준으로 하였다.

교육목표에 포함된 의미단위, 그룹, 범주, 주제의 수를 비교 한 결과는 Table 4와 같다. 설립 주체와 학년별 정원, 학제에 따라서는 통계적 차이가 없었으나, 지리적 위치가 수도권인 경우, 연구중심병원을 보유한 경우에는 그렇지 않은 경우에 비해 교육목표에 포함된 의미단위, 그룹, 범주, 주제의 수가 유의하게 많았다.

44 개의 범주와 13 개의 주제에 대한 하위집단 간 차이를 확 인하기 위하여 Pearson chi-square test를 수행하였다. 유의 수준 0.05 에서 통계적으로 유의한 범주 및 주제는 Table 5 와 같다. 국립 의과대학이 사립 의과대학에 비하여 '신앙정신과 ‘인류수준기여' 범주 보유 비율이 유의하게 낮았으며, 학년별 
Table 4. Comparison of Meaning Units, Groups, Categories, and Themes between Subgroups

\begin{tabular}{|c|c|c|c|c|c|c|c|c|c|}
\hline \multirow{2}{*}{\multicolumn{2}{|c|}{ Characteristics of the medical schools }} & \multicolumn{2}{|c|}{ Meaning units } & \multicolumn{2}{|c|}{ Groups } & \multicolumn{2}{|c|}{ Categories } & \multicolumn{2}{|c|}{ Themes } \\
\hline & & Mean \pm SD & $p$-value ${ }^{\text {al }}$ & Mean \pm SD & p-value $^{\text {al }}$ & Mean \pm SD & p-value $^{\text {al }}$ & Mean $\pm S D$ & $p$-value ${ }^{\text {al }}$ \\
\hline \multirow{2}{*}{$\begin{array}{l}\text { Subject of } \\
\text { foundation }\end{array}$} & Private (31) & $21.45 \pm 7.89$ & 0.169 & $17.09 \pm 5.24$ & 0.355 & $15.03 \pm 4.17$ & 0.360 & $8.42 \pm 2.02$ & 0.491 \\
\hline & National (10) & $17.70 \pm 5.16$ & & $15.40 \pm 3.94$ & & $13.70 \pm 3.09$ & & $7.90 \pm 2.13$ & \\
\hline \multirow[t]{2}{*}{ Size of a class } & 51 or more (23) & $18.73 \pm 6.15$ & 0.094 & $15.91 \pm 3.96$ & 0.294 & $14.17 \pm 3.22$ & 0.334 & $8.00 \pm 2.17$ & 0.305 \\
\hline & 50 or fewer (18) & $22.83 \pm 8.45$ & & $17.66 \pm 6.00$ & & $15.38 \pm 4.71$ & & $8.67 \pm 1.84$ & \\
\hline \multirow{2}{*}{$\begin{array}{l}\text { Graduate entry } \\
\text { program }\end{array}$} & Adopted (partly or fully) (27) & $21.85 \pm 7.26$ & & $17.59 \pm 4.76$ & 0.104 & $15.40 \pm 3.84$ & 0.116 & $8.59 \pm 2.09$ & 0.195 \\
\hline & Not adopted (14) & $18.00 \pm 7.37$ & & $14.92 \pm 5.06$ & & $13.35 \pm 3.91$ & & $7.71 \pm 1.85$ & \\
\hline \multirow{2}{*}{$\begin{array}{l}\text { Geographic } \\
\text { location }\end{array}$} & Nonmetropolitan (26) & $18.34 \pm 6.63$ & 0.011 & $15.26 \pm 4.75$ & 0.015 & $13.57 \pm 3.72$ & 0.014 & $7.65 \pm 1.89$ & 0.007 \\
\hline & Metropolitan (15) & $24.33 \pm 7.44$ & & $19.13 \pm 4.48$ & & $16.66 \pm 3.63$ & & $9.40 \pm 1.84$ & \\
\hline \multirow{2}{*}{$\begin{array}{l}\text { Research-driven } \\
\text { hospital affiliation }\end{array}$} & Not affiliated (32) & $18.96 \pm 6.11$ & 0.009 & $15.56 \pm 4.39$ & 0.005 & $13.84 \pm 3.56$ & 0.007 & $7.94 \pm 1.89$ & 0.034 \\
\hline & Affiliated (9) & $26.11 \pm 9.36$ & & $20.66 \pm 5.07$ & & $17.77 \pm 3.86$ & & $9.56 \pm 2.12$ & \\
\hline
\end{tabular}

${ }^{a}$ t-test between two subgroups of medical schools grouped by five criteria (subject of foundation, size of a class, graduate entry program, geographic location, and research-driven hospital affiliation).

Table 5. Comparison of Themes and Categories between Subgroups

\begin{tabular}{|c|c|c|}
\hline $\begin{array}{l}\text { Characteristics of the } \\
\text { medical schools }\end{array}$ & Themes $^{\mathrm{a}}$ & Categories $^{\text {a) }}$ \\
\hline Subject of foundation & None & $\begin{array}{l}\text { Religious spirit (national<private) } \\
\text { Human-kind-level contribution (national< }<\text { private) }\end{array}$ \\
\hline Size of a class & Respect for life (51 or more $<50$ or fewer) & None \\
\hline Graduate entry program & None & Lifelong learning (not adopted<adopted) \\
\hline Geographic location & $\begin{array}{l}\text { Problem-solving ability } \\
\text { (nonmetropolitan }<\text { metropolitan) } \\
\text { Creativity (nonmetropolitan }<\text { metropolitan) }\end{array}$ & $\begin{array}{l}\text { Primary healthcare (metropolitan }<\text { nonmetropolitan) } \\
\text { Creativity (nonmetropolitan }<\text { metropolitan) }\end{array}$ \\
\hline $\begin{array}{l}\text { Research-driven hospital } \\
\text { affiliation }\end{array}$ & Creativity (not affiliated<affiliated) & $\begin{array}{l}\text { Values (not affiliated<affiliated) } \\
\text { International-level contribution (not affiliated < affiliated) } \\
\text { Healthcare manager (not affiliated < affiliated) } \\
\text { Self-reflection (not affiliated<affiliated) } \\
\text { Creativity (not affiliated < affiliated) }\end{array}$ \\
\hline
\end{tabular}

${ }^{a} \chi^{2}$ between two subgroups of medical schools grouped by five criteria (subject of foundation, size of a class, graduate entry program, geographic location, and research-driven hospital affiliation).

정원이 50명 이하인 의과대학에서 '생명존중' 주제 보유 비율 이 유의하게 높았다. 또한 의전원 제도를 도입한 대학에서 '평 생학습 범주 보유 비율이 유의하게 높았다. 수도권 소재 의과 대학은 비수도권 소재 의과대학보다 '창의성' 범주와 ‘문제해 결능력' 주제 보유 비율이 유의하게 높으나 '일차의료' 범주 보 유 비율은 유의하게 낮았다. 마지막으로 연구중심병원을 보 유한 의과대학의 경우 '가치관, '국제수준기여', '보건의료관리 자, '자기성찰능력', ‘창의성' 범주 보유 비율이 연구중심병원 미보유 의과대학보다 유의하게 높았다.

\section{4. "한국의 의사상"과의 비교}

귀납적 내용분석을 통해 도출한 범주와 주제를 “한국의 의 사상”에 대응시킨 결과는 Table 6과 같다. 44개 범주는 "한국 의 의사상"에 제시된 5 개 영역에 고루 분포하였으며, 16 개의 역량 중 12 개의 역량에 적어도 하나 이상의 범주가 대응되었 다. 이들 중 가장 많은 수의 의과대학 교육목표에 포함된 역량 은 영역 1 의 ' 1 . 의학 지식 및 임상 술기' 역량으로 38 개 의과 대학에서 이를 다루고 있었다. 반면, 영역 1의 '3. 환자 안전', 
Table 6. Correspondences between Competencies of the "Korean Doctor's Role" and the Identified Categories

\begin{tabular}{|c|c|c|c|}
\hline \multirow{2}{*}{ Domain } & \multirow{2}{*}{$\begin{array}{c}\text { Korean doctor's role } \\
\text { Competency }\end{array}$} & \multicolumn{2}{|l|}{ Educational purposes } \\
\hline & & Categories $\left(n^{a}\right)$ & $n^{b)}$ \\
\hline \multirow[t]{3}{*}{ 1. Patient care } & 1. Medical knowledge and clinical skills & $\begin{array}{l}\text { Biomedical knowledge (33) } \\
\text { Medical skills (30) } \\
\text { Problem-solving ability (17) } \\
\text { Ability to making a diagnosis and provide treatment (17) }\end{array}$ & 38 \\
\hline & 2. Professional attitudes & $\begin{array}{l}\text { Attitudes (14) } \\
\text { Personality (13) } \\
\text { Expertise (12) }\end{array}$ & 28 \\
\hline & 3. Patient safety & & 0 \\
\hline \multirow[t]{4}{*}{$\begin{array}{l}\text { 2. Communication } \\
\text { and cooperation }\end{array}$} & 1. Communication and cooperation with patients & $\begin{array}{l}\text { Patient-centered care (17) } \\
\text { Trust (3) }\end{array}$ & 19 \\
\hline & $\begin{array}{l}\text { 2. Communication and cooperation with patient } \\
\text { family }\end{array}$ & & 0 \\
\hline & $\begin{array}{l}\text { 3. Communication and cooperation with } \\
\text { colleagues }\end{array}$ & Teamwork among health professions (7) & 7 \\
\hline & 4. Communication and cooperation with society & & 0 \\
\hline \multirow[t]{3}{*}{$\begin{array}{l}\text { 3. Social } \\
\text { accountability }\end{array}$} & 1. Maintaining health and wellbeing & $\begin{array}{l}\text { Community-level contribution (24) } \\
\text { National-level contribution (22) } \\
\text { Health promotion (10) }\end{array}$ & 35 \\
\hline & $\begin{array}{l}\text { 2. Participating in healthcare policy } \\
\text { decision-makingprocess and dealing with } \\
\text { future society }\end{array}$ & $\begin{array}{l}\text { Leadership (24) } \\
\text { Cope actively with changes (14) } \\
\text { Future-oriented (8) } \\
\text { Healthcare manager (2) }\end{array}$ & 33 \\
\hline & $\begin{array}{l}\text { 3. Disaster relief and promoting international } \\
\text { cooperation }\end{array}$ & $\begin{array}{l}\text { Service spirit (17) } \\
\text { International level contribution (13) }\end{array}$ & 26 \\
\hline \multirow[t]{4}{*}{ 4. Professionalism } & 1. Patient care based on ethics and autonomy & $\begin{array}{l}\text { Ethics and morality (31) } \\
\text { Self-reflection (9) }\end{array}$ & 32 \\
\hline & 2. Doctor-patient relationship & Being cultured (humanities and social sciences) (6) & 6 \\
\hline & 3. Self-regulation led by professionals & - & 0 \\
\hline & 4. Professionalism and self-management & $\begin{array}{l}\text { Professionalism (4) } \\
\text { Good physical health (1) }\end{array}$ & 5 \\
\hline \multirow[t]{2}{*}{$\begin{array}{l}\text { 5. Education and } \\
\text { research }\end{array}$} & 1. Education & $\begin{array}{l}\text { Lifelong learning (34) } \\
\text { Ability of education (6) }\end{array}$ & 34 \\
\hline & 2. Research & $\begin{array}{l}\text { Research ability (28) } \\
\text { Reasoning ability (7) }\end{array}$ & 28 \\
\hline \multirow[t]{2}{*}{$\begin{array}{l}\text { Not corresponded } \\
\text { categories }\end{array}$} & Does not exist in Korean doctor's role & $\begin{array}{l}\text { Human-kind-level contribution (21) } \\
\text { Creativity (20) } \\
\text { Founding philosophy/ideals of education (19) } \\
\text { Primary healthcare (18) } \\
\text { Respect for life (15) } \\
\text { Love for mankind (12) } \\
\text { Religious spirit (10) } \\
\text { Advance towards various areas of medicine (10) } \\
\text { Wisdom (1) } \\
\text { Self-realization (1) }\end{array}$ & \\
\hline & Embracive terms & $\begin{array}{l}\text { Sense of duty (11) } \\
\text { Social accountability (10) } \\
\text { Respect for diversity (10) } \\
\text { Cooperation (9) } \\
\text { Communication (8) } \\
\text { Values (5) }\end{array}$ & \\
\hline
\end{tabular}

${ }^{a \mid}$ The number of medical schools that contains the category in their educational goals or purposes, ${ }^{b /}$ The number of medical schools that contains the competency in their educational goals or purposes. 
영역 2의 '2. 환자 보호자와의 소통과 협력' '4. 사회와의 소통 과 협력', 영역 4의 ‘3. 전문직 주도의 자율 규제’ 역량에는 적 절히 대응되는 범주를 찾지 못하였다. 역량에 대응되지 못하 고 남은 범주는 16 개였는데, 적절한 대응을 이루는 역량 또는 세부역량이 부재한 경우가 10 개, 역량 또는 세부역량에 대응 시키기에는 의미가 포괄적이어서 ‘영역' 수준 대응이 적절한 경우가 6개였다.

\section{고찰}

본 연구에서는 귀납적 내용분석방법을 활용하여 41 개 의과 대학/의전원 교육목표를 총체적, 공시적으로 분석함으로써 현재 한국 기본의학교육이 지향하는 바를 살펴보고자 하였다. 또한 설립 주체, 학년별 정원, 학제, 지리적 위치, 연구중심병 원 보유 여부 등에 따라 의과대학을 나누고 하위그룹 간 교육 목표의 차이를 확인해보았다. 마지막으로 이러한 결과를 최 근 발표된 "한국의 의사상"에 대응시킴으로써 기본의학교육 의 현 방향과 사회적으로 요구되는 의사상을 서로 비교해보 고자 하였다.

41 개 의과대학/의전원의 교육목표를 의미단위 수준에서 보 았을 때, 평균적으로는 20.54개의 의미단위를 포함하고 있었 지만, 가장 많은 학교는 39 개의 의미단위를, 가장 적은 학교 는 6개의 의미단위만을 포함하는 것으로 나타나 의과대학 간 차이가 작지 않음을 확인할 수 있었다. 이러한 차이는 주제 수 준에서도 유지되었는데, 평균적으로는 8.29개의 주제를 포함 하고 있었지만, 가장 많은 학교는 13 개 주제를 모두 포괄한 반면 가장 적은 학교는 4 개의 주제만을 포함하는 것으로 확인 되었다. 현실적으로 4년 혹은 6년으로 제한된 의과대학 교육 과정에서 의사로서 필요한 역량을 모두 다루기에는 한계가 있기에 모든 의과대학에서 모든 주제와 범주를 다루는 것이 반드시 바람직한 것은 아니다. 또한 교육목표를 기술하는 방 식은 각 의과대학이 자신이 기본의학교육에서 추구하는 특성 을 드러내는 수단이기도 하다. 그러나 어느 의과대학을 졸업 하든 의과대학을 졸업한 학생은 일정 수준의 역량을 갖출 수 있어야 하며, 대중에게 공개되는 의과대학 공식 문서로서 교 육목표가 지니는 사회적 책무성을 종합적으로 고려한다면[4],
모든 의과대학이 공통된 형식을 갖추지는 않더라도 최소한 적절한 양의 구체적 단어를 사용하여 교육목표의 의미와 맥 락을 명확히 하는 것은 중요할 것이다.

최종적으로 도출된 13 개 주제 중 12 개 주제는 최소 19 개 이 상의 의과대학 교육목표에서 다루고 있었으며, '의(醫) 전문 성, '전문직업성', '사회 여러 층에 대한 기여', '자기관리 및 개 발은 35 개 이상의 의과대학 교육목표에 포함되어 있었다. 반 면, '교육능력'은 단 여섯 개 의과대학 교육목표에서만 다루고 있었는데, '교육”이 “한국의 의사상”의 다섯 번째 영역인 '5. 교 육과 연구 내에 독립적인 역량으로 구분되어 있을 뿐 아니라, 교육, 연구, 진료로 대표되는 의과대학과 대학병원의 세 가지 핵심 기능 중 하나라는 점에서[1] 상대적으로 과소평가되고 있다고 볼 수 있다. 만일 '교육'의 의미를 '의과대학에서의 교 수-학생 간 교육'으로 제한한다면 '교육능력'은 기본의학교육 보다는 졸업 후 의학교육, 연수교육 등을 통해 의과대학교수 로서의 진로를 추구하는 일부 학생에게만 강조하는 것으로 충분하다 볼 수 있다. 그러나 의과대학에서의 학생동료 간 교 육(peer-teaching), 대학병원 전공의로서 후배 전공의 및 학 생을 대상으로 담당하게 될 교육, 의사가 환자, 보호자, 일반 인에 대해 수행하는 교육 등이 모두 '교육능력'에 내포된다면, '교육'은 기본의학교육에서도 한층 강조되어야 할 필요가 있 을 것이다.

범주 중 빈도가 높은 것은 ‘평생학습’이었으며, '의과학지식', ‘윤리 및 도덕성, ‘의학술기', '연구능력' 등이 뒤를 이었다. 반 면 '보건의료관리자, '신체적 건강', '자아실현, '지혜', '신뢰', '전문직업성'은 4 개 이하 의과대학에서만 다루고 있었다. 이 중 ‘자아실현', '지혜', '신뢰', '전문직업성'은 그 의미가 상대적 으로 추상적이거나 포괄적이어서 다른 범주와 중첩될 가능성 이 있다. 한편 “보건의료관리자'는 “한국의 의사상”에도 '3. 사 회적 책무성' 영역 하위에 ‘보건의료정책 결정 참여 및 미래 대응 역량의 한 요소로 포함되어 있지만, 기본의학교육보다 는 졸업 후 의학교육과 연수교육에서 다루는 것이 더 효과적 일 수 있다. 그러나 자신의 건강에 대한 관리는 "한국의 의사 상” 중 ‘4. 전문직업성' 영역 중 ‘전문직업성과 자기 관리’ 역량 의 세부 역량으로 포함되어 있을 뿐 아니라, 의과대학에 입학 했을 당시에 비해서 입학 이후에 주관적으로 인지하는 건강 수준이 저하된다는 기존 연구 결과에 비추어 볼 때[17], '신체 
적 건강은 기본의학교육에서부터 다룰 필요가 있어 보인다.

설립 주체, 학년별 정원, 학제, 지리적 위치, 연구중심병원 보유 여부 등에 따라 의과대학 하위집단을 나누어 교육목표 의 차이를 확인해보았다. 그 결과 설립 주체, 학년별 정원, 의 전원 도입 여부에 따라서 의미단위, 그룹, 범주, 주제의 수에 는 차이가 없었으며, 범주와 주제의 구체적 항목에서도 한두 개의 차이만이 확인되었다. 이러한 결과에 대하여 국내 의과 대학이 설립 주체, 규모, 학제와 무관하게 서로 대등하거나 균 일한 교육을 지향하고 있다는 점을 긍정적으로 바라볼 수 있 다. 그러나 마찬가지로 각 의과대학의 차이와 개성이 충분히 교육에 반영되고 있지 않다는 지적 역시 가능하다. 이는 국내 의학교육이 사립 주체 주도로 이뤄지고 있는 배경과 학년 당 50명 이하의 소규모 의과대학이 모두 1985년 이후에 설립된 배경이 의학적 혹은 교육학적 원리보다는 정치경제적 원리에 영향을 받았다는 기존에 제기된 비판과도 유사하다[11,18].

반면 하위집단 간 특징이 구분되어 나타난 분류도 있었다. 수도권에 위치한 경우 그렁지 않은 경우보다 교육목표에 포 함된 의미단위, 그룹, 범주, 주제의 수가 유의하게 많았고, 그 중 수도권에 위치한 대학은 ‘창의성', ‘문제해결능력' 주제 포 함 비율이, 비수도권에 위치한 대학은 '일차의료' 범주 포함 비율이 유의하게 높았다. 즉, 비수도권 의과대학은 졸업생에 게 일차의료를 통한 지역사회에의 기여를 강조하는 반면, 수 도권 의과대학 창의적 연구를 통한 문제해결을 중요시 할 것 으로 기대할 수 있다. 비록 상관관계의 강도는 크지 않으나 '일차의료' 범주 포함 여부와 '지역사회수준 기여' 범주 포함 여부의 상관관계(r=0.346, $\mathrm{p}=0.027)$ 와, '창의성' 주제 포함 여 부와 '연구능력' 주제 포함 여부의 상관관계(r=0.350, p= $0.025)$ 가 통계적으로 유의하다는 점도 이러한 해석을 일부 지 지한다고 볼 수 있다.

또한 연구중심병원을 보유한 의과대학의 경우에도 그렇지 않은 경우보다 의미단위, 그룹, 범주, 주제의 수가 유의하게 많았으며, 세부적으로는 '가치관, '국제수준기여', '보건의료관 리자, ‘자기성찰능력' ‘창의성' 범주의 비율이 유의하게 높았 다. 앞에서와 마찬가지로 ‘창의성' 주제 포함 여부와 '연구능력' 주제 포함 여부가 통계적으로 유의한 정적 상관을 가진다는 점을 고려하면, 연구중심병원을 보유한 의과대학은 기본의학 교육에서도 '자기성찰'과 '창의성'을 바탕으로 '국제수준의 기
여를 할 수 있는 '연구역량 개발이라는 지향점을 가질 것이라 기대할 수 있다.

마지막으로 “한국의 의사상”과의 비교 결과 5 개 영역에 걸 쳐 고르게 범주가 대응됨을 확인할 수 있었다. 그러나 16 개 역량을 개별적으로 살펴보면, ' 1 . 환자 진료’ 영역의 ‘환자 안 전' 역량, '2. 소통과 협력' 영역의 ‘환자 보호자와의 소통과 협 력, '사회와의 소통과 협력' 역량, '4. 전문직업성' 영역의 '전문 직 주도의 자율 규제 '역량에 대응되는 범주가 부재하였다. 이 중 ‘1. 환자 진료’ 영역의 ‘환자 안전' 역량은 임상 직무를 본격 적으로 수행하는 졸업 후 의학교육 이후에 다루는 것이 보다 적합할 수 있다. 한편, '2. 소통과 협력' 영역은 미대응된 '상호 다양성존중, ‘의사소통', '협력' 등의 범주가 포괄적 수준에서 소통과 협력과 관련된 역량을 다루고 있다고 볼 수 있다. 반면 '4. 전문직업성' 영역의 '전문직 주도의 자율 규제' 역량은 그 중요도에 비추어 볼 때 현재 기본의학교육에서 조금 더 강조 될 필요가 있어 보인다. '자율규제'는 '임상적 자율, '직무윤리' 와 더불어 전문직업성의 3 대 요소이며, 이들 요소는 각각 독 립된 것이 아닌, 서로 연관되어 상호 공존하는 가치에 가깝기 때문이다[19]. 또한 현 기본의학교육에서는 '임상적 자율'의 토대가 될 '의(醫) 전문성'을 거의 모든 의과대학에서 지향하 고 있으며, '직무윤리' 역시 30 개 이상의 의과대학이 '윤리 및 도덕성'을 강조하며 추구하고 있다. 따라서 이처럼 다른 두 가 지 요소가 다수 의과대학에서 다뤄지고 있는 만큼, 세 요소의 적절한 균형을 위하여 기본의학교육에서 '전문직 주도의 자율 규제'에도 더 많은 시간을 투자할 필요가 있어 보인다.

본 연구의 한계점을 짚어보면 다음과 같다. 첫째, 분석 대상 에 포함된 '교육목적'과 '교육목표'가 한 의과대학의 교육 지향 점을 온전히 반영하지 못했을 수 있다. 예를 들어, 교육목표가 주기적, 지속적으로 개정될 수 있는 문서임을 고려하면, 자료 수집 시점에 해당 의과대학이 실제로 사용하고 있는, 혹은 가 장 최근에 개정한 교육목표가 아직 반영되지 않았을 수도 있 다. 또한 분석 대상을 정의하기 위하여 '포함 기준과 '제외 기 준을 적용하는 과정에서 해당 의과대학 전체의 '비전'이나 ‘미션, 교육 지향점을 표명하기 위해 부가적으로 공지된 ‘핵심 가치, '교육이념' 등 일부 문헌이 배제되었고, 상세한 설명을 위한 '해설' 또는 '설명과 같은 주석이 제외되었다. 그러나 대 부분의 의과대학은 '포함 기준과 '제외 기준'을 적용한 후에도 
교육목적과 교육목표 전문(솣)이 포함되었기 때문에, 41 개 의과대학/의전원에 공통적인 기준을 적용함으로써 모든 의과 대학 교육목표를 거의 동등한 수준에서 분석할 수 있었다는 긍정적 측면도 있다.

둘째, 교육목표의 단어나 문장은 매우 함축적이기에 동일 한 단어도 서로 다른 의미로 기술되었을 수 있다. 비단 '가치 관이나 ‘태도'와 같이 그 자체가 다양한 요소로 구성되어 있는 단어뿐 아니라, '봉사, '신뢰’, '협력'과 같이 행위의 대상이 다 양할 수 있는 단어도 맥락에 따라 서로 다른 의미를 가질 수 있다. 연구진은 이러한 한계를 인지하고, 분석과정에서 원문 의 맥락을 고려하며, 단어의 의미가 불분명하거나 의견의 불 일치가 있을 경우 반복적 논의와 합의과정을 거쳤으나 이러 한 한계를 모두 극복했다고 보기는 어렵다.

이에 따르는 본 연구의 세 번째 한계는 도출한 범주 혹은 주제에 다양한 해석의 여지가 존재한다는 것이다. 범주를 구 성하는 것은 질적 내용분석의 핵심 특징이며, 상호배타성 (mutually exclusive)과 완결성(exhaustive)이 요구된다[14]. 이 중 완결성은 본 연구에서 분석대상으로 포함한 모든 문장 의 의미단위를 코딩함으로써 어느 정도 달성할 수 있었으나, 상호배타성은 인간 체험(human experience)의 본질상 모든 경우에 가능한 것은 아니며[14], 이는 의사의 다양한 역량에 대해서도 마찬가지일 것이다. 따라서 본 연구에서 도출된 범 주와 주제들은 서로 완벽하게 상호배타적이라 보기 어렵고, 관점을 달리 해가며 다양한 해석을 할 수 있다.

마지막으로 교육목표와 관련하여 반드시 던져보아야 할 질 문이 있는데, 바로 대내외적으로 표명한 교육목표가 실제 성 과로 연결되는지에 대한 것이다[4,20]. 즉, 의과대학 교육목 표가 진정한 의미를 갖기 위해서는 실제 교육현장에서 교육 목표를 염두에 둔 교육이 이루어져야 하며, 더 나아가 그러한 교육이 졸업생의 역량으로 이어지는 것이 중요하다. 그러나 본 연구와 같이 교육목표 그 자체에 대한 연구만으로는 이러 한 부분을 간접적으로 평가하는 데 그칠 수 있다.

미래 의사 양성의 첫 단계인 기본의학교육을 통해서 개인 과 사회의 요구에 부응할 수 있는 역량을 배양하기 위해서는, 적절한 교육목적과 교육목표 설정이 선행되어야 한다. 본 연 구에서는 국내 41 개 의과대학/의전원의 교육목표를 총체적, 공시적으로 분석함으로써 현 한국 기본의학교육의 방향을 살
펴보았으며, 나아가 이를 “한국의 의사상”과 비교하고 차이를 확인해 보았다. 다수 의과대학이 성과바탕교육으로 이행하고 있는 현 시점에서, 여러 의과대학이 각자의 특색을 살리면서 도, 미래 의사로서 갖추어야 할 기본적 역량을 배양시킬 수 있 는 교육과정 개발과 개선에 본 연구 결과가 유용하게 이용될 수 있기를 기대한다.

Acknowledgements: None.

Funding: None.

Conflicts of interest: None.

\section{REFERENCES}

1. Ramsey PG, Miller ED. A single mission for academic medicine: improving health. JAMA 2009; 301: 14751476.

2. Gordon VN, Grites TJ, Habley WR; National Academic Advising Association. Academic advising: a comprehensive handbook. 2nd ed. San Francisco, USA: Jossey-Bass; 2008. p 229-241.

3. Leithwood KA, Hallinger P. Second international handbook of educational leadership and administration. Dordrecht, The Netherlands: Springer Netherlands; 2002. p 9-40.

4. Lewkonia RM. The missions of medical schools: the pursuit of health in the service of society. BMC Med Educ 2001; 1: 4.

5. Roh H, Rhee BD, Lee JT, Bae SK. Development of task-based learning outcomes according to clinical presentations for clinical clerkships. Korean J Med Educ 2012; 24: 31-37.

6. Hur Y, Lee K. Are medical students being properly cared for? A question for the current student advisory program. Korean J Med Educ 2013; 25: 221-228.

7. Na BJ, Kang J, Kim JY, Yun J, Han S, Hwang W, Hur 
Y. What do faculties need most in a faculty development program? Korean J Med Educ 2014; 26: 137-141.

8. Morphew CC, Hartley M. Mission statements: a thematic analysis of rhetoric across institutional type. J Higher Educ 2006; 77: 456-471.

9. Medical College Accreditation Standards [Internet]. Korean Institute of Medical Education and Evaluation; 2012 [cited 2014 December 30]. Available from: http://www.kimee.or.kr/new2009/download/평가인증기 준.hwp.

10. History of Korea Research-Driven Hospitals Project [Internet]. Korea Health Industry Development Institute; 2013 [cited 2014 December 30]. Available from: http://rndhospital.khidi.or.kr/board?menuId=MENU003 87.

11. Ahn D. The future roles of Korean doctors: cultivating well-rounded doctors. Korean Med Educ Rev 2014; 16 : 119-125.

12. Kern DE, Thomas PA, Howard DM, Bass EB. Curriculum development for medical education: a six step approach. Baltimore, USA: Johns Hopkins University Press; 1998. p 5-7.

13. Dang JY, Choy YS, Kim YH. A study of Mission statements for strategic management: focusing on the tertiary care hospitals in Korea and special functioning hospitals in Japan. Korean J Hosp Manage 2013; 18: 70-87.

14. Elo S, Kyngäs $H$. The qualitative content analysis process. J Adv Nurs 2008; 62: 107-115.

15. Graneheim UH, Lundman B. Qualitative content analysis in nursing research: concepts, procedures and measures to achieve trustworthiness. Nurse Educ Today 2004; 24: 105-112.

16. Lee YH, Lee YM, Kim BS. Content analysis of standardized-patients' descriptive feedback on student performance on the CPX. Korean J Med Educ 2010; 22: 291-301.

17. Paro HB, Morales NM, Silva CH, Rezende CH, Pinto RM, Morales RR, Mendonça TM, Prado MM. Health-related quality of life of medical students. Med Educ 2010; 44: 227-235.

18. Lee MS. Uidae sinseol nonliwa injeungpyeongga jedo: jibanguidae sinseolui munje. Uiryojeongchaekporeom 2009; 7: 84-97.

19. Ahn D. Development of medical professionalism in South Korea. J Korean Med Assoc 201 1; 54: 1137-1145.

20. Bart CK, Hupfer M. Mission statements in Canadian hospitals. J Health Organ Manag 2004; 18: 92-110. 